\title{
Tendinopatías: etiología, histopatología y avances terapéuticos
}

\section{Review article}

Tendinopathies: etiology, histopathology and therapeutic advances

Pág. 14-23

Francisco Bonilla Alvarado ${ }^{1}$

1.Fisioterapeuta, estudiante de la maestría académica en ciencias morfológicas.

Docente del departamento de Ciencias Morfológicas de la Universidad de Ciencias Médicas (UCIMED), San José Costa Rica

Fisioterapeuta de Clínica de Fisioterapia de la Universidad de Ciencias Médicas (UCIMED), San José Costa Rica

Recibido: 20/11/19

Aprobado: 29/11/19

\section{Resumen:}

Objetivos: Describir los principales cambios histopatológicos presentes en las tendinopatías, su patogénesis y los avances terapéuticos encontrados en la literatura.

Palabras Claves: Tendinopatías, histología, patogénesis, inflamación, tendinosis, regeneración.

Materiales y métodos: Se realizó una búsqueda de literatura relacionada con las tendinopatías su patogénesis, cambios histológicos, avances terapéuticos en el uso de células madres, factores de crecimiento (plasma rico en plaquetas) y terapia farmacológica. Se utilizaron las bases de datos de pubmed, clinical key, science direct. Se incluyeron artículos de revisiones bibliográficas entre 2005 al 2018, escritos en inglés.

Conclusiones: Las tendinopatías tienen un complejo origen multifactorial, donde los factores extrínsecos e intrínsecos juegan un papel importante para el desarrollo de dicha afectación. La inflamación tiene un rol determinante en el desarrollo de la patogenia de las tendinopatías. La terapia farmacológica antiinflamatoria tiene efectos a corto plazo en la disminución del dolor, sin embargo, resulta contraproducente a largo plazo. Existe controversia sobre el uso del Plasma rico en plaquetas (PRP) y de terapia celular (stem cells), se requiere de mayor investigación que pueda evidenciar la eficacia de dichos tratamientos.

\section{Abstract}

Objective: To describe the main histopathological changes present in the tendinopathies, the pathogenesis and the therapeutic advances found in the literature on treatments with stem cells, growth factors (platelet-rich plasma) and pharmacological treatment.

Methods: a literature search related to pathogenesis, histological changes of tendinopathies and therapeutic advances regarding the use of cellular therapies (stem cells), therapy with growth factors (platelet-rich plasma) and pharmacological therapy. The bibliographic search was carried out in the Pubmed databases, Clinical Key, Science Direct. Bibliographic review articles were used between 2005 and 2018, written in English.

Conclusions: Tendinopathies have a complex multifactorial origin, where extrinsic and intrinsic factors play an important role in the development of this affectation. Inflammation plays a decisive role in the development of the pathogenesis of tendinopathies. Pharmacological antiinflammatory therapy has short-term effects on pain reduction, however, it is counterproductive in the long term. There is controversy about the use of PRP and cell therapy (stem cells); more research is needed to demonstrate the effectiveness of this treatments in tendinopathies. 


\section{Introducción}

El tendón es una estructura fibrosa que permite que el músculo esquelético se inserte en el hueso lo que permite lograr la transmisión de fuerza para que se genere locomoción. Histológicamente, es un tejido conjuntivo denso regular, que se caracteriza porque sus fibras de colágeno están agrupadas muy juntas y organizadas en haces - fascículos de manera paralela, y hay poca presencia de matriz extracelular (MEC). 1 Contiene células llamadas tendinocitos, que consisten en fibroblastos rodeados de MEC especializada que separa cada fibrilla colágena de sostenes. El tendón se encuentra cubierto por una capa de tejido conectivo muy delgado que es denominado el epitendón. Además, se encuentra formado por fascículos separados por el endotendón, que contiene pequeños vasos sanguíneos y nervio. ${ }^{1}$

Los tendones reciben inervación, principalmente sensorial, de las fibras nerviosas circundantes en el músculo o la piel. El tejido del peritendón es ricamente inervado por terminaciones nerviosas libres, que funcionan con receptores nociceptivos. Otras fibras nerviosas penetran a través de las vainas de tejido conjuntivo hasta la superficie del tendón, y finalizan en las terminaciones nerviosas sensitivas, las cuales son consideradas como órganos sensitivos de la coordinación, control motor y mediación de dolor. ${ }^{2}$ El aporte vascular se localiza en la unión miotendinosa, unión osteotendinosa y peritendón, por lo tanto, se puede entender que la vascularización de un tendón maduro es muy pobre, por lo que al ser un tejido poco vascularizado tiene limitadas en las medidas de reparación ante las tendinopatías.

La tendinopatía es un término usado para describir una condición de tendón doloroso con confirmación de alteración y hallazgos microscópicos y macroscópicos. Resulta ser un problema clínico común y conlleva un impacto en la calidad de vida de las personas. ${ }^{2}$ Su etiología es de origen multifactorial, y no se comprende del todo bien. ${ }^{3}$ Se han descritos distintos factores de riesgo, a menudo en dos principales grupos: factores extrínsecos (los que actúan sobre el cuerpo) e intrínsecos (los que actúan desde dentro del cuerpo). ${ }^{3}$

Entre los factores extrínsecos se pueden mencionar los movimientos de sobreuso, algunos deportes, como por ejemplo, los deportes con carga de peso en el miembro inferior y los deportes aéreos como la natación y el voleibol que tienden a producir tendinopatías del hombro. También los deportes que transcurren con movimientos de alta exigencia de almacenamiento de energía y sobrecarga tensional como los saltos del básquetbol, voleibol, corredores cuesta arriba, los cambios de dirección repentinos y los que requieren patear en terrenos de campo. ${ }^{4}$ Milliaras y O'neil $^{5}$ describen otros factores extrínsecos como los estiramientos repetitivos cortos cíclicos de la unidad músculotendinosa (correr, saltos), desequilibrios de compresión y tensión del tendón, así como cambios en las cargas del entrenamiento deportivo, entre otros.

Los factores intrínsecos pueden ser modificables o no, y se relacionan con la composición corporal, el estilo de vida y la salud general del individuo. ${ }^{4}$ Se han descrito factores de riesgo intrínsecos asociados a la genética, principalmente en las implicaciones de las alteraciones de dos variantes para el gen COL5A1 y el gen tenascina-C (TNC). El gen COL5A1 participa en la codificación del colágeno tipo 5 y en la alineación y organización del colágeno tipo I, lo cual predispone a mayor vulnerabilidad a las tendinopatias. ${ }^{4}$ Por otra parte, TNC es una proteína anti adhesiva, que es importante en la regulación de la capacidad de carga y compresión del tendón. Alteraciones de dicha proteína se han relacionado con la aparición de tendinopatías aquileas. ${ }^{4}$

Algunos autores ${ }^{4,6}$ mencionan los factores sexo y la edad. Por ejemplo las mujeres posmenopáusicas exhiben mayor tasa de tendinopatías. ${ }^{6} \mathrm{El}$ aumento de la edad se asocia con cambios en la actividad celular, propiedades mecánicas, función muscular, y disminución de la resistencia así como del potencial de curación inherente. ${ }^{4,5}$ Además, se han descrito factores sistémicos asociados a la tendinopatías como la obesidad, diabetes mellitus tipo II, dislipidemias, fumado y entesopatías 


\section{Materiales y métodos}

Se realizó una búsqueda de artículos científicos en las bases de datos de Pubmed, Science Direct, Clinical Key con las palabras claves: "tendón", "tendon histology", "tendinopathy", "tendinitis", "Tendon repair". Se incluyen solamente artículos en idioma español e inglés, pertenecientes al periodo comprendido entre el año 2005 al año 2018.

\section{Cambios histológicos del tendón}

La tendinosis es un término utilizado para describir los cambios morfológicos y estructurales descritos en tendinopatías crónicas,con o sin presencia de sintomatología en los pacientes, así como lo muestran estudios histopatológicos de rupturas espontaneas de tendones. . $^{2,6}$

Los hallazgos patológicos más comunes asociados con la tendinopatía no insercional son la tendinosis y la paratendinopatía. ${ }^{7}$ La tendinosis comprende cambios patológicos como la desorganización y separación de las fibras de colágeno, el aumento de la sustancia fundamental mucoide, la hipercelularidad y la neovascularización, en su mayoría sin signos de inflamación intratendinosa. ${ }^{7}$ Sin embargo, existe controversia en este detalle debido a que se han publicado estudios que describen la existencia de una fase inflamatoria inicial $^{7-10}$

El principal componente de los tendones es el colágeno tipo I, correspondiendo al $65-80 \%$, mientras que dentro de la matriz extracelular, hay presencia de tenoblastos, tendinocitos (90-95\% del componente celular), condrocitos, células vasculares, células sinoviales y células de musculo liso $(5-10 \%) .{ }^{7}$ Entre los cambios presentes en las tendinosis se encuentra una desorganización y separación de las fibras de colágeno tipo I además, un aumento de la presencia de colágeno tipo III, esto acrecienta el riesgo de rupturas y lesiones debido a que este tipo de colágeno tiene menor resistencia mecánica que el tipo I, predisponiendo a posibles rupturas tendinosas. ${ }^{7-11}$ Por otra parte, hay incremento de proteoglicanos, glicosaminoglicanos y acumulación de agua dando un aspecto macroscópico grisáceo, amorfo e hinchado. ${ }^{4,7}$

Se han descrito distintas manifestaciones celulares, dependiendo de las fases o estadios de la tendinosis, por ejemplo se puede encontrar estados de hipercelularidad de tendinocitos, con cambios en la forma del núcleo más redondeas y con aumento de producción de proteoglicanos y proteínas. Sin embargo, se han descrito también estadios de hipocelularidad, asociados a apoptosis.

Por otra parte, se dan cambios en la irrigación del tejido, debido a la neovascularización, por lo general, asociada a procesos crónicos de degeneración. $^{2}$ La angiogénesis se asocia a la liberación de neuroquímicos ligados a la inflamación neurogénica. ${ }^{2}$ La angiogénesis se ve mediada por marcadores angiogénicos del factor de crecimiento endotelial vascular (VEGF) y el factor de von Willebrand (VWF). ${ }^{2} \mathrm{El}$ VEGF se considera un mediador que permite la permeabilidad vascular, tiene un rol importante en la inflamación. Además, se presenta en zona de neurogénesis, ligadas a la secreción de sustancia $P$, sustancia trasmisora de dolor. ${ }^{2}$

\section{Reparación y regeneración}

El mantenimiento de la estructura del tendón está influenciada por las estructuras de la MEC, dicha matriz es regulada por los tendinocitos que se encargan de mantener la homeostasis del tendón. ${ }^{4} \mathrm{El}$ tendinocito es capaz de responder a las cargas mecánicas por medio de cambios en la MEC, tanto por la formación o degradación de la misma. La preservación de dicha homeostasis es esencial en el proceso de reparación tisular. ${ }^{4} \mathrm{La}$ carga fisiológica normal del tendón es necesaria para el mantenimiento adecuado de la MEC. Se han descrito que posibles causas de afectaciones agudas de un tendón pueden ser originadas por un deterioro crónico, sin embargo, otros autores mencionan que la afectación aguda puede ser generada por un evento traumático o por una única sobrecarga del tendón, mientras que la afectación crónica se debe a microtraumatismos por repetición. ${ }^{4}$ 
Ante una lesión del tendón, se inicia su proceso de reparación. Este es descrito en tres principales fases: fase inflamatoria, fase proliferativa y fase de remodelación. ${ }^{4,12}$ Se comienza la reparación por medio de la fase inflamatoria, la cual inicia con la migración de plaquetas, células inflamatorias neutrófilos, eritrocitos, monocitos y macrófagos. ${ }^{12}$

\section{Sustancias vasomotoras promueven la} permeabilidad vascular, la angiogénesis y el reclutamiento de más células, con esto, hay liberación de distintos factores de crecimiento $y$ citoquinas. El Factor de necrosis tumor- $\alpha$ (TNF- $\alpha$ ), IL-1 $\beta$, IL-6 e IL-8 ejercen acciones proinflamatorias, la IL-6, IL-8, IL-10 tienen efectos antiinflamatorios. La IL-6 tiene dualidad en su función, con efectos inflamatorios y de síntesis de colágeno. ${ }^{12}$ Esta fase tiene una duración desde el inicio de la lesión hasta 7 días. ${ }^{4,7,12}$

La fase proliferativa se caracteriza por un incremento de fibroblastos en el MEC y por la presencia de factores de crecimiento que inducen la neovascularización y quimiotaxis de los fibroblastos, de manera que se estimula la proliferación y la síntesis de colágeno tipo III, esta etapa tiene una duración de unas 6 semanas. ${ }^{4}$

Posteriormente, la etapa de remodelación, genera una reorganización de las fibras colágenas, además, se produce un recambio de colágeno tipo III por colágeno tipo I. La MEC seguirá madurando con una cantidad creciente colágeno, el volumen del callo tendinoso disminuirá y su fuerza biomecánica continuará mejorando. ${ }^{4} \mathrm{La}$ maduración del colágeno y su remodelación inicia desde la tercer semana y puede continuar hasta un año después de ocurrida la lesión. ${ }^{6}$

\section{Patogénesis de las tendinopatías}

La homeostasis del tendón está regulada por la carga mecánica, la actividad celular, las vías neurales y los mediadores celulares. Por tanto, hay que recalcar que la patogénesis del tendón resulta ser un proceso multifactorial. Como se describió anteriormente, los factores intrínsecos y extrínsecos influencian en la aparición de patología del tendón. Los cambios primarios ocurren en el tipo de colágeno, en el proceso inflamatorio o en la combinación de ambas.

\subsection{Mediación inflamatoria}

Como se ha descrito, la tendinopatía es el resultado de un proceso degenerativo del tendón, en el cual la inflamación tiene importancia en estadios iniciales. Ante microtraumatismo presentes en el tendón, se secretan distintas sustancias que promueven la reparación, incluyendo mediadores inflamatorios. ${ }^{9}$

Debido a que la inflamación está vinculada con el proceso degenerativo, este puede iniciarse por la inflamación y de igual manera, la inflamación tiene efectos sobre la degeneración tardía. ${ }^{9}$

Anteunalesiónaguda, se puede encontrarprocesos de inflamación inicial mediados por la liberación de citoquinas, factores inmunoreguladores, proliferación celular, degradación de la matriz extracelular, necrosis y dolor. ${ }^{12}$ La inflamación se cree que tiene el rol de iniciar de manera más temprana los cambios fibróticos y degenerativos. ${ }^{12}$

También está vinculada con la expresión de mediadores inflamatorios por el tendinocito, como los son citoquinas pro inflamatorias, antiinflamatorias, factores de crecimiento, como lo son: TNF- $\alpha$ (Factor de necrosis tumoral- $\alpha$ ), IL$1 \beta$ (Interleucina-1 $\beta$ ), IL-6, IL-10, VEGF, TGF- $\beta$ (Factor de crecimiento transformante- $\beta$ ), COX-2 (ciclooxigenasa) y PGE-2 (Prostaglandina E2). ${ }^{12}$

El IL-1 $\beta$ es un mediador importante en los procesos de infección o lesión. Este es inducido por macrófagos, monocitos, y hoy en día se ha evidenciado que puede ser dado por algunas células del tejido conectivo. ${ }^{9} \mathrm{IL}-1 \beta$ induce a los tendinocitos a la liberación de mediadores como COX-2, PGE-2, MMP-1 (Metaloproteinasa de la matriz extrcelular-1), que pueden acelerar la degradación de la MEC. 8,12 Además, la IL-1 $\beta$ regula de manera significativa la producción de colágeno tipo I en la MEC del tendón a nivel del ARNm, por lo que se puede ver una deposición reducida en las tendinopatías. ${ }^{12}$ 
La IL-1b facilita la liberación de la sustancia, esto asociado a la contribución inflamatoria y la respuesta nociceptiva de las tendinopatias. ${ }^{12}$

La IL-6 tiene una función inmunoregulatoria en la curación del tendón, se ha evidenciado la presencia de citoquinas IL-6 en tendinopatías. ${ }^{9}$ Además, se ha asociado con la síntesis de colágeno y un incremento de la expresión de marcadores procolágeno en el peritendón, esto en muestras de tendón calcáneo humano. ${ }^{9}$

La interleucina-17A (IL-17A) es un mediador proinflamatorio que pertenece a la familia IL-17 y está asociado con la destrucción y degeneración del tejido. Se ha encontrado influencia de IL17-A en la síntesis de colágeno y en la mediación inflamatoria de TNF-a, IL-6, and IL-8. Además, se ha asociado al proceso de apoptosis de los tendinocitos. ${ }^{9}$

El TNF- $\alpha$ se asocia a las tendinopatías y su degeneración. El TNF- $\alpha$ activa los tendinocitos para producir más citoquinas proinflamatorias, antiinflamatorias como IL-1 $\beta$, TNF-a, IL-6 and IL-10 y enzimas degenerativas de MEC. Estas enzimas inhiben la síntesis de colágeno tipo I. Además, se ha asociado que el TNF- $\alpha$ en conjunto con la caspasa-3 genera un proceso pro apoptosis. ${ }^{9,12}$ 5.2

\section{Mediación celular}

La presencia de células inmunes en las tendinopatías ha sido un tema controversial. Algunos estudios han descrito que no existe presencia de dichas células en las tendinopatías, sin embargo, otros lo contradicen y resaltan su mediación durante el proceso de patogénesis del tendón, particularmente en las etapas iniciales. ${ }^{8,9}$, 12,13

Estudios recientes han mostrado la infiltración de células inflamatorias tipo macrófagos. ${ }^{8,13}$ Estos tienen un papel esencial en procesos inflamatorios y de reparación de tejidos. La vías que producen la activación de los macrófagos (M1 y M2) están sustentadas por interferón de activación de macrófagos, NF-kB, STAT6 y vías de activación de receptores de glucocorticoides, los cuales han sido identificados en tendinopatías del manguito rotador. ${ }^{8}$ En etapas tempranas de las tendinopatías, se notaron expresiones de genes y proteínas mediadas por NF-kB, mientras que en procesos crónicos, se encontró la expresión de genes y proteínas mediadas por STAT6 y vías de activación de receptores de glucocorticoides. Esto puede indicar que existen distintos mecanismos que pueden cambiar dependiendo del estadio de las tendinopatías. 8

Diferentes estudios identifican que los mastocitos están presentes en la tendinopatias.9 Dichas células juegan un papel importante en procesos inflamatorios y se han encontrado en zonas rodeando los microvasos, gracias a técnicas por inmunomarcaje específico. 9, 14

Los tendinocitos, son las células de mayor abundancia en los tendones, encargadas de la secreción de la MEC, que en situaciones de patogénesis, aumenta el número de células en comparación con un tendón normal. Los tendinocitos pueden sintetizar diversas citoquinas como el TNF- $\alpha$, IL-1 $\beta$, IL-6, IL-10 y el factor de crecimiento endotelial vascular (VEGF). ${ }^{9,}{ }^{15}$ Los tendinocitos se vuelven metabólicamente más activos por la influencia de las citoquinas, como parte del proceso inflamatorio. ${ }^{9}$

\subsection{Inflamación neurogénica}

La sustancia $\mathrm{P}(\mathrm{SP})$ es un neuropéptido potente que se asocia a la producción de dolor y está relacionado con las tendinopatías. A este tipo de inflamación se le conoce como neurogénica..$^{9}$ Este neuropéptido facilita la liberación de histamina de los mastocitos y promueve la vasodilatación y extravasación de las células inmunitarias ${ }^{12}$

La presencia de niveles significativos de SP, glutamato, y péptido relacionado con el gen de la calcitonina (CGRP) proveen un mecanismo de la mediación del dolor en tendinopatías crónicas. ${ }^{2}$ Esto se pudo evidenciar en tendinopatías crónicas como el codo de tenista, donde se reportó niveles aumentados de manera significativa de CGRP, SP y glutamato. ${ }^{2}$ Además, se considera que SP está involucrado con la mediación del dolor, edema y fibrosis tendinosa como parte del proceso degenerativo. ${ }^{9}$ 


\subsection{Angiogénesis y aporte vascular}

La neoangiogénesis va estar regulada por la hipoxia, la sobrecarga mecánica y por las sustancias neovasogénicas. Una de las principales sustancias es el VEGF, que induce la migración de las células endoteliales en la zona de la lesión, por medio de quimiotaxis y vasodilatación. ${ }^{7}$ Por otra parte, VEGF , IL-6, IL-21R y demás citoquinas, tienen un potente estímulo para la expresión de metaloproteinasas de la MEC en distintas células (tendinocitos, fibroblastos, condrocitos, células endoteliales), que desempeñan un rol en el proceso degenerativo del tendón. ${ }^{12}$ La neovascularización es considerada como una contribución al deterioro de las propiedades mecánicas del tendón. ${ }^{7}$

\section{Avances en terapéuticos}

Las tendinopatías suelen ser un problema que común en la población a nivel mundial, se estima que afectan alrededor de 30 millones de personas anualmente. ${ }^{16}$ Actualmente, el manejo de las tendinopatías puede ser realizarse de manera conservadora o quirúrgica. Conservadoramente se trata con farmacológicamente como corticoesteroides en inyectables, medicamentos antiinflamatorios no esteroides, terapia física y terapia de ondas de choque extracorpóreas. ${ }^{16}$

Sin embargo, su evolución resulta lenta y hay probabilidades de que en algunos casos no se logre rehabilitar completamente. Por otra parte, el tratamiento quirúrgico es utilizado generalmente en casos agudos severos o en rupturas tendinosas importantes. La reparación quirúrgica con injertos es la opción terapéutica de uso actual, que por lo general conlleva a limitaciones de la movilidad, por la formación de cicatriz, y de adherencias, aumentando el riesgo de rupturas. ${ }^{16}$ Por esta razón, hay gran necesidad de buscar opciones terapéuticas que logren recuperar al tendón en su morfología, biomecánica y funcionalidad. La terapia biológica ha puesto su interés en el tratamiento de la regeneración de tejidos musculoesqueléticos. Se considera que con el descubrimiento de nuevas vías de señalización y linajes celulares se puede favorecer la reparación y regeneración de distintos tejidos.

\subsection{Terapia de células madre (stem cells)}

Los avances en el uso terapéutico de las células madre han mostrado tener potencial para ser utilizados en la reparación y regeneración de tejidos dentro de ellos el tendón. Las células madre pueden ser clasificadas según su potencial de diferenciación, en células pluripotenciales como las de origen embriológico (ESC) y las células pluripotenciales inducidas (IPSC) que pueden diferenciarse en cualquier tipo de células. Por otra parte, las células mutilpotenciales, como las células mesequimales (MSC), pueden convertirse en más de un tipo de células, sin embargo, su potencial de diferenciación sigue siendo menor que el de las pluripotenciales. ${ }^{16}$

Estudios han mostrado que el uso de células madre embriológicas se han diferenciado en células mesenquimales sobre gel de fibrina implementadas en un tendón de rata, resultando en mejoras en la estructura y mecánica de este..$^{16}$ Por otra parte, se ha descrito que el uso de células embriológicas diferenciadas en células mesenquimales han causado formación ectópica en tendones curados, ${ }^{4}$ lo cual hace necesarias mayores investigaciones en este tema.

Las MSC pueden diferenciarse en una variedad de tipos de células de tejido conectivo, incluidos los tendinocitos. Las MSC pueden derivarse de diversas fuentes de tejido, como lo son la médula ósea (BMSC), el tendón (TPSC) y el tejido adiposo (ADSC). Estudios en animales han demostrado que las MSC tienen la capacidad de mejorar la reparación del tendón. ${ }^{16}$

Estudios han identificado mejoras histológicas tras inyecciones de MSC de sangre periférica en tendones de ovejas inducidos a lesión por colagenasa. ${ }^{16}$ Además, la administración alogénica de MSC de sangre de codón umbilical en una tendinopatía del flexor superficial de los dedos mejoró su desempeño de curación y fuerza, evaluado por ultrasonografía. ${ }^{16}$ También se han realizado combinaciones de células madre, buscando mejorar el efecto terapéutico. 
El uso de BMSC y TPSC fueron utilizados con la administración de láminas celulares con esta combinación, en tendón patelar de rata, dando mejoras en la reparación del tejido en comparación a la utilización de una sola fuente celular. ${ }^{16}$

A pesar de distintos avances en la terapia celular, existen ciertas preocupaciones con respeto a la seguridad de dichas administraciones. Se han descrito alteraciones como la formación ósea ectópica y tumores tras el uso de BMSC y ADSC. Por otra parte, el uso de ESC y IPSC han evidenciado riesgo de formación de teratomas tras su administración..$^{16}$ Las TDSC han tomado particular interés debido a que tienen la capacidad de promover la curación del tendón sin aumentar el riesgo de condrosificación ectópica4, sin embargo, la obtención de TSPC pueden conducir a la morbilidad del sitio donante, por lo que resulta un desafío conseguir una muestra de calidad para su utilización. ${ }^{16} \mathrm{En}$ modelos experimentales animales, la mayoría de ensayos clínicos refieren la necesidad de crear un protocolo de tratamiento estándar para poder identificar la opción celular más adecuada y el tipo específico de reparación de las tendinopatías ${ }^{9,16}$ además de su observación a largo plazo para evidenciar si el comportamiento del tejido será similar al del tendón nativo. ${ }^{4}$

\subsection{Terapia con factores de crecimiento}

Dentro del proceso inicial de reparación del tendón, la regulación de la expresión de algunos factores de crecimientos es beneficiosa para la reparación del tejido. Se pueden mencionar el factor de crecimiento de fibroblastos básico (BFGF), factores de crecimiento tipo insulina 1 (IGF-1), factor de crecimiento derivado de plaquetas (PDGF), factor de crecimiento transformante- $\beta$ (TGF $\beta$ ) y VEGF. ${ }^{9}$

El Plasmo rico en plaquetas se define como una preparación con altas concentraciones de plaquetas. ${ }^{17}$ El papel funcional del PRP se debe a la presencia de factores de crecimiento y citoquinas que se encuentran en las plaquetas, que se pueden administrar localmente en tejidos poco vascularizados y que son importantes su reparación. ${ }^{17}$
Actualmente en la medicina ortopédica y del deporte, se han tomado algunos criterios para el uso del PRP en tendinopatías, entre ellos se pueden mencionar:

1) Dolor por más de 3 a 6 meses y mayor a 4 en la escala visual analógica del dolor (0-10). 2) Confirmación clínica e imagenológica de tendinopatía u osteoartritis.

3) Persistencia de los síntomas posterior a las terapias conservadoras (anti inflamatorios no esteroideos, fisioterapia).

4) Evasión de una cirugía.

5) Adhesión al tratamiento de varias sesiones de inyecciones de al menos 6 semanas. ${ }^{17}$

La aplicación de PRP en la epicondilitis lateral (codo de tenista) ha demostrado mejorías en la capacidad funcional y alivio de dolor, mejorando la estructura, esto valorado mediante imagenología médica en comparación al uso de inyecciones de corticoesteroides. ${ }^{17}$ Sin embargo, al corto plazo, los corticoesteroides tienen mejor resultado para disminuir el dolor.

En tendinopatías aquileas, se recomienda el PRP como una terapia de segunda línea, ya que se ha reportado que no existen diferencias significativas entre su uso y el agua salina. 17,20 Por otra parte, en las tendinopatías patelares, no se han desarrollado estudios controlados aleatorizados que concluyan sobre el uso de PRP vs terapias conservadoras, por lo que este podría ser utilizado en casos resistentes a la terapia conservadora. ${ }^{17}$

Otros autores ${ }^{19}$ mencionan que el uso del PRP mejora la regeneración, disminuye el dolor y ayuda en la capacidad funcional del hombro en personas con desgarros del manguito rotador. Además, se menciona que el PRP pobre en leucocitos mejora la curación del tendón con mejores resultados histológicos, por lo que se considera como una mejor medida para el tratamiento clínico de la tendinopatía en comparación con el PRP rico en leucocitos. 9 Muchos de estos estudios concluyen que existe controversia en el uso del PRP ${ }^{9,20}$ por lo que se recomienda mayor investigación en su utilización ${ }^{17}$ que permita medir sus resultados a 
corto, mediano y largo plazo.

\subsection{Tratamiento farmacológico}

Los medicamentos orales antiinflamatorios no esteroides (AINES) han sido utilizados por décadas como tratamiento en las tendinopatías, sin embargo su uso aun genera controversia. 9 Algunos estudios han mostrado que la medicación oral logra ser efectiva en el control de dolor a corto plazo, sin mejoramiento de la función de la extremidad afectada. ${ }^{4}$

Bibliografía reciente considera que los AINES pueden proveer efectos negativos a largo plazo en el proceso de regeneración de los tejidos. Esto asociado a complicaciones gastrointestinales, renales y cardiovasculares. ${ }^{4}$ Las inyecciones de corticoesteroides también son debatidas por su controversia. Se discute que al igual que la medicación oral, los inyectables de corticoides generan un efecto de alivio del dolor a corto plazo, sin embargo, a largo plazo puede generar aumento de los síntomas. Se ha reportado disminución de la fuerza del tendón en estudios realizados en animales, y se ha descrito ruptura tendinosa posterior a la inyección de corticoides en humanos. ${ }^{7}$

El uso de medicamentos de moléculas pequeñas ha tomado mayor interés debido a que actúan sobre vías de señalización específicas en la fisiología y función celular. Las moléculas pequeñas pueden modular la transducción de señalización y la transcripción de genes. ${ }^{21}$ Además, estos medicamentos pueden ser consumidos oralmente, permitiendo una absorción directa, también son fáciles de sintetizar, purificar y producir en cantidades masivas. ${ }^{21}$

Algunos de las vías de señalización identificadas en las tendinopatías se encuentran: la Vía de señalización de NF-kB. Medicación de moléculas pequeñas pueden suprimir la vía de NF-kB, eliminando mediadores inflamatorios asociados a las tendinopatías, entre algunos de los agentes mencionados se encuentran el resveratrol and cúrcuma. El resveratrol se asocia a la supresión de la IL-1 $\beta$, mientras que la cúrcuma puede inhibir la expresión de MMP, COX-2, Bax y Caspasa-3, los cuales ya están asociados con la estimulación de señalización NF-kB. ${ }^{21}$

Por otra parte, la señalización de la proteína BMP (Proteína morfogénica ósea) puede regular la homeostasis de diferentes tejidos, sin embargo, aún no está claro su efecto en el tendón. El inhibidor específico del receptor I de BMP (LDN193189) puede inhibir eficazmente la formación de osificación heterotópica en un modelo animal, esto ha permitido que se pueda pensar en utilizarse para redirigir las TSPC (Tendon stem/ progenitor cells) a una diferenciación tenogénica para mejorar la cicatrización del tendón. 21 Aun estos acercamientos resultan algo incipientes, por lo que Investigaciones adicionales de vías de señalización intracelular nos proporcionarían más dianas terapéuticas alternativas para mantener la homeostasis del sistema musculoesquelético.

\section{Conclusiones}

Con la revisión de las distintas fuentes, es claro que la tendinopatías tiene un complejo origen multifactorial, donde los factores extrínsecos e intrínsecos juegan un papel importante para el desarrollo de dicha afectación. Esta afectación puede llegar a ser incapacitante, y su evolución puede ser de un par de semanas, hasta convertirse en un problema crónico de meses o años. La recuperación puede ser compleja, derivar en recaídas o terminar como una ruptura tendinosa. Nuevos avances han logrado vincular procesos inflamatorios con la evolución de la patogénesis de las tendinopatías.

Los mediadores inflamatorios tienen diferentes funciones, por lo que se pueden superponer y actuar como sinergistas en las tendinopatias.9 Estos tienen la capacidad de modular la estructura y composición de la MEC y a su vez, las células inflamatorias cambian el fenotipo y la función de las células tendinosas. 
Los tendinocitos como las células inmunes pueden producir mediadores inflamatorios, sin embargo, no está claro cuál de estas células inician el proceso. $^{9}$

Tratamientos actuales para las tendinopatías no han logrado generar un impacto satisfactorio para la recuperación de esta afectación. Esto pone en discusión la efectividad del tratamiento quirúrgico y farmacológico.

El uso de tratamientos antiinflamatorios como lo son los glucocorticoides y los AINES han sido considerados poco efectivos o contraproducentes a largo plazo. ${ }^{4,7,9}$ Autores ${ }^{9}$ mencionan que la terapia antiinflamatoria debe ser enfocada desde el diseño de la biología molecular, de manera que las moléculas pequeñas puedan modificar algunas vías de señalización de interés en el proceso de la tendinopatía.

Las terapias biológicas celulares y factores de crecimiento deben ser sometidos a una mayor investigación, para proveer resultados sobre su efectividad y alcances.

La terapia con células madres como medida terapéutica en la medicina regenerativa ha generado en los últimos años, datos interesantes relacionadas con la regeneración del tendón. Sin embargo, la mayoría de textos consultados concluyen que la información actual es escaza, lo que hace necesario mayor estudio en este tema con el fin de identificar cuáles son las mejores fuentes celulares, las más favorables y efectivas en la regeneración del tendón.

\section{Conflictos de interés}

No se reporta ningún tipo de conflicto de interés. El autor no tenía afiliaciones profesionales o financieras que sesgaron este trabajo.

\section{(c) $(1) \Theta(9)$}




\section{Referencias bibliográficas}

1.Pawlina, W. Ross Histología Texto y Atlas: Correlación con biología molecular y celular. (7 ed). Barcelona: Wolter Kluwer; 2016.

2.Fedorczyk JM. Tendinopathies of the elbow, wrist, and hand: histopathology and clinical considerations. $J$ Hand Ther. 2012 Apr-Jun;25(2):191-200. doi: 10.1016/j. jht.2011.12.001.

3.Federer AE, Steele JR, Dekker TJ, Liles JL, Adams SB. Tendonitis and Tendinopathy: What Are They and How Do They Evolve? Foot Ankle Clin. 2017 Dec;22(4):665-676. doi: 10.1016/j.fcl.2017.07.002.

4.Gian Luigi Canata, Pieter d'Hooghe, Kenneth J. Hunt Muscle and tendon injuries.USA: Springerlink, 2017.

5.Malliaras P, O'Neill S. Potential risk factors leading to tendinopathy. Apunts Med Esport. 2017;52(194):71-77. Doi: http://dx.doi.org/10.1016/j.apunts.2017.06.001

6.Miller M, Thompson S. DeLee \& Drez's Orthopaedic Sports Medicine (4ed.). USA: Elsevier, 2015.

7.Physiology and Pathophysiology of Musculoskeletal Tissues

8.Gomes M, Reis RL, Rodrigues M. Tendon Regeneration: Understanding Tissue Physiology and Development to Engineer Functional Substitutes. San Diego: Elsevier, S. A; 2015.

9.Dean BJF, Dakin SG, Millar NL, Carr AJ. Review: Emerging concepts in the pathogenesis of tendinopathy. Surgeon. 2017 Dec;15(6):349-354. doi: 10.1016/j. surge.2017.05.005.

10.Tang C, Chen Y, Huang J, Zhao K, Chen X, Yin Z, Heng BC, Chen W, Shen W. The roles of inflammatory mediators and immunocytes in tendinopathy. J Orthop Translat. 2018 Apr 14;14:23-33. doi: 10.1016/j. jot.2018.03.003.

11.Dakin SG, Dudhia J, Smith RK. Resolving an inflammatory concept: the importance of inflammation and resolution in tendinopathy. Vet Immunol Immunopathol. 2014 Apr 15;158(3-4):121-7. doi: 10.1016/j.vetimm.2014.01.007.

12.Brown MN, Shiple B, Scarpone M. Physical Medicine and Rehabilitation Clinics of North America, Volume 27, Issue 4, November 2016, Pages 941-984. Regenerative Approaches to Tendon and Ligament Conditions.
13.D'Addona A, Maffulli N, Formisano S, Rosa D. Inflammation in tendinopathy. Surgeon. 2017 Oct;15(5):297-302. doi: 10.1016/j.surge.2017.04.004.

14.Dean BJ, Gettings P, Dakin SG, Carr AJ. Are inflammatory cells increased in painful human tendinopathy? A systematic review. $\mathrm{Br} \mathrm{J}$ Sports Med. 2016 Feb;50(4):216-20. doi: 10.1136/ bjsports-2015-094754.

15.Scott A, Lian O, Bahr R, Hart DA, Duronio V, Khan $\mathrm{KM}$. Increased mast cell numbers in human patellar tendinosis: correlation with symptom duration and vascular hyperplasia.Br J Sports Med 2008;42:753-7.

16.Pufe T, Petersen WJ, Mentlein R, Tillmann BN. The role of vasculature and angiogenesis for the pathogenesis of degenerative tendons disease. Scand J Med Sci Sports. 2005 Aug;15(4):211-22.

17.Liu L, et al., Advances of stem cell based-therapeutic approaches for tendon repair. Journal of Orthopaedic Translation (2017), http://dx.doi.org/10.1016/j. jot.2017.03.007

18. Hadley CJ, Shi WJ, Murphy $\mathrm{H}$, Tjoumakaris FP, Salvo JP, Freedman KB. The Clinical Evidence Behind Biologic Therapies Promoted at Annual Orthopaedic Meetings: A Systematic Review. Arthroscopy. 2018 Nov 16. pii: S0749-8063(18)30474-2. doi: 10.1016/j. arthro.2018.05.037.

19.Martínez-Martínez A, Ruiz-Santiago F, GarcíaEspinosa J. Plasma rico en plaquetas: ¿mito o realidad? Radiologia. 2018 Nov - Dec;60(6):465-475. doi: 10.1016/j.rx.2018.08.006.

20. Hurley ET, Lim Fat D, Moran CJ, Mullett H. The efficacy of platelet-rich plasma and platelet-rich fibrin in arthroscopic rotator cuff repair: a meta-analysis of randomized controlled trials. Am J Sports Med. 2018

21.Andia I, Maffulli N. Clinical Outcomes of Biologic Treatment for Chronic Tendinopathy. Operative techniques in ortophaedics. http://dx.doi.org/10.1053/j. oto.2015.12.007

22.Chen $Y$, Huang J, Tang $C$, Chen $X$, Yin $Z$, Heng BC, Chen W, Shen W. Small molecule therapeutics for inflammation-associated chronic musculoskeletal degenerative diseases: Past, present and future. Exp Cell Res. 2017 Oct 1;359(1):1-9. doi: 10.1016/j. yexcr.2017.07.027.. 